\title{
A new iterative method based on the modified proximal-point algorithm for finding a common null point of an infinite family of accretive operators in Banach spaces
}

\author{
T.M.M. Sow \\ Amadou Mahtar Mbow University, \\ Dakar Senegal \\ sowthierno89@gmail.com
}

\begin{abstract}
In this paper, we introduce and study a new iterative method for finding a common null point of an infinite family of accretive operators with a strongly accretive and Lipschitzian operator, by using the proximal-point algorithm. And also we prove that the common null point is a unique solution of variational inequality without imposing any compactness-type condition on either the operators or the space considered. Finally, some applications of the main results to equilibrium problems and fixed point problems with an infinite family of pseudocontractive mappings are given. The main result is a generalization and improvement of numerous well-known results in the available literature.
\end{abstract}

\section{RESUMEN}

En este artículo, introducimos y estudiamos un nuevo método iterativo para encontrar un cero común de una familia infinita de operadores acretivos con un operador Lischitziano fuertemente acretivo, usando el algoritmo punto-proximal. También demostramos que el cero común es la única solución de una desigualdad variacional sin imponer ninguna condición de tipo compacidad en ninguno de los operadores o los espacios considerados. Finalmente, se entregan algunas aplicaciones de los resultados principales a problemas de equilibrio y problemas de punto fijo con una familia infinita de aplicaciones pseudo-contractivas. El resultado principal es una generalización y mejora de numerosos resultados bien conocidos en la literatura disponible.

Keywords and Phrases: Proximal-point algorithm; Accretive operators; Variational inequality; Common zeros.

2020 AMS Mathematics Subject Classification: 46T05; 47H06; 47H09; 47H10.

\section{(cc) BY-NC}

(C)2020 by the author. This open access article is licensed under a Creative Commons Attribution-NonCommercial 4.0 International License. 


\section{Introduction}

Let $H$ be a real Hilbert space and $K$ be a nonempty subset of $H$. For a set-valued map $A: H \rightarrow 2^{H}$, the domain of $A, D(A)$, the image of a subset $S$ of $H, A(S)$ the range of $A, R(A)$ and the graph of $A, G(A)$ are defined as follows:

$$
\begin{aligned}
& D(A):=\{x \in H: A x \neq \emptyset\}, A(S):=\cup\{A x: x \in S\}, \\
& R(A):=A(H), G(A):=\{(x, u): x \in D(A), u \in A x\} .
\end{aligned}
$$

A multi-valued map $A: D(A) \subset H \rightarrow 2^{H}$ is called monotone if the inequality

$$
\langle u-v, x-y\rangle \geq 0
$$

holds for each $x, y \in D(A), u \in A x, v \in A y$. A single-valued operator $A: K \rightarrow H$ is said to be strongly positive bounded linear if there exists a constant $k>0$ such that

$$
\langle A x, x\rangle \geq k\|x\|^{2}, \quad \forall x, y \in K \text {. }
$$

Remark 1. It is immediate that if $A$ is $k$-strongly positive bounded linear, then $A$ is $k$-strongly monotone and $\|A\|$-Lipschitz continuous.

A monotone operator $A$ is called maximal monotone if its graph $G(A)$ is not properly contained in the graph of any other monotone operator. It is well known that $A$ is maximal monotone if and only if $A$ is monotone and $R(I+r A)=H$ for all $r>0$ and $A$ is said to satisfy the range condition if $\overline{D(A)} \subset R(I+r A)$. Many problems arising in different areas of mathematics, such as optimization, variational analysis and differential equations, can be modeled by the equation

$$
0 \in A x
$$

where $A$ is a monotone mapping. The solution set of this equation coincide to a null points set of A. Such operators have been studied extensively (see, e.g., Bruck Jr [5], Chidume [9], Rockafellar [29], Xu [30] and the references therein).

Consider, for example, the following: let $f: H \rightarrow \mathbb{R} \cup\{\infty\}$ be a proper lower semi continuous and convex function. The subdifferential, $\partial f: H \rightarrow 2^{H}$ of $f$ at $x \in H$ is defined by

$$
\partial f(x)=\left\{x^{*} \in H: f(y)-f(x) \geq\left\langle y-x, x^{*}\right\rangle \quad \forall y \in H\right\} .
$$

It is easy to check that $\partial f: H \rightarrow 2^{H}$ is a monotone operator on $H$, and that $0 \in \partial f(x)$ if and only if $x$ is a minimizer of $f$. Setting $\partial f \equiv A$, it follows that solving the inclusion $0 \in A u$, in this case, is solving for a minimizer of $f$. 
In order to find a solution of problem (1.1), Rockafellar 29] introduced a powerful and successful algorithm which is recognized as Rockafellar proximal- point algorithm: for any initial point $x_{0} \in H$, a sequence $\left\{x_{n}\right\}$ is generated by:

$$
x_{n+1}=J_{r_{n}}\left(x_{n}+e_{n}\right), \forall n \geq 0,
$$

where $J_{r}=(I+r A)^{-1}$ for all $r>0$, is the resolvent of $A$ and $\left\{e_{n}\right\}$ is an error sequence in a Hilbert space. In the recent years, the problem of finding a common element of the set of solutions of convex minimization, variational inequality and the set of fixed point problems in real Hilbert spaces, Banach spaces and complete CAT(0) (Hadamard) spaces have been intensively studied by many authors; see, for example, [20, 21, 19, 29, 30] and the references therein.

Very recently, Eslamian and Vahidi [10] introduced a new iterative method base on proximal point algorithm with strongly positive bounded linear operator for solving a system of inclusion problem. They established a strong convergence theorem which extends the corresponding results in $30,2,132,28,13,14,15,16,16,17,18$.

Theorem 2 (Eslamian and Vahidi [10). Let $H$ be a real Hilbert space and $K$ be a nonempty, closed and convex subset of $H$. Let $\left\{B_{i}\right\}, i \in \mathbb{N}^{*}:=\{1,2,3, \ldots\}$ be an infinite family of operators of $H$ such that $\bigcap_{i=1}^{\infty} B_{i}{ }^{-1}(0) \neq \emptyset$ and $\bigcap_{i=1}^{\infty} \overline{D\left(B_{i}\right)} \subset K \subset \bigcap_{i=1}^{\infty} R\left(I+r B_{i}\right)$, for all $r>0$. Let $A: H \rightarrow H$ be a $k$-strongly bounded linear operator with a coefficient $\bar{\gamma}$ and $f$ be a $b$-contraction mapping of $K$ into itself with a constant $b \geq 0$.

Let $\left\{x_{n}\right\}$ be a sequence defined iteratively from arbitrary $x_{0} \in K$ by:

$$
\left\{\begin{array}{l}
y_{n}=\beta_{n, 0} x_{n}+\sum_{i=1}^{\infty} \beta_{n, i} J_{r_{n}}^{B_{i}} x_{n} \\
x_{n+1}=\alpha_{n} \gamma f\left(x_{n}\right)+\left(I-\alpha_{n} A\right) y_{n} .
\end{array}\right.
$$

Let $\left.\left\{r_{n}\right\} \subset\right] 0, \infty\left[,\left\{\beta_{n, i}\right\}\right.$ and $\left\{\alpha_{n}\right\}$ be real sequences in $(0,1)$ satisfying:

(i) $\lim _{n \rightarrow \infty} \alpha_{n}=0 ; \quad$ (ii) $\sum_{n=0}^{\infty} \alpha_{n}=\infty, \quad \sum_{i=0}^{\infty} \beta_{n, i}=1$,

(iii) $\lim _{n \rightarrow \infty} \inf r_{n}>0$, and $\lim _{n \rightarrow \infty} \inf \beta_{n, 0} \beta_{n, i}>0$, for all $i \in \mathbb{N}$.

Assume that $0<\gamma<\frac{\bar{\gamma}}{b}$. Then, the sequence $\left\{x_{n}\right\}$ generated by (1.2) converges strongly to $x^{*} \in$ $\bigcap_{i=1}^{\infty} B_{i}^{-1}(0)$

Above discussion yields the following questions.

Question 1:Can results of Eslamian and Vahidi [10], and so on be extended from Hilbert spaces to Banach spaces? 
Question 2: We know that Lipschitzian mapping is more general than contraction. What happens if the contraction is replaced by Lipschitzian mapping ?

Question 3: We know that $k$-strongly accretive operators and $L$-Lipchizian operators is more general than the strong positive bounded linear operators. What happens if the strongly positive bounded linear operators is replaced by $k$ - strongly accretive operators and $L$-Lipchizian operators?

The purpose of this paper is to give affirmative answers to these questions mentioned above. Applications are also included to valide our new findings.

\section{Preliminairies}

Let $E$ be a real Banach space and $C$ be a nonempty, closed and convex subset of $E$. We denote by $J$ the normalized duality map from $E$ to $2^{E^{*}}$ ( $E^{*}$ is the dual space of E) defined by:

$$
J(x):=\left\{x^{*} \in E^{*}:\left\langle x, x^{*}\right\rangle=\|x\|^{2}=\left\|x^{*}\right\|^{2}\right\}, \forall x \in E .
$$

Let $S:=\{x \in E:\|x\|=1\} . E$ is said to be smooth if

$$
\lim _{t \rightarrow 0^{+}} \frac{\|x+t y\|-\|x\|}{t}
$$

exists for each $x, y \in S$. E is said to be uniformly smooth if it is smooth and the limit is attained uniformly for each $x, y \in S$.

Let $E$ be a normed space with $\operatorname{dimE} \geq 2$. The modulus of smoothness of $E$ is the function $\rho_{E}:[0, \infty) \rightarrow[0, \infty)$ defined by

$$
\rho_{E}(\tau):=\sup \left\{\frac{\|x+y\|+\|x-y\|}{2}-1:\|x\|=1,\|y\|=\tau\right\} ; \quad \tau>0 .
$$

It is known that a normed linear space $E$ is uniformly smooth if

$$
\lim _{\tau \rightarrow 0} \frac{\rho_{E}(\tau)}{\tau}=0 .
$$

If there exists a constant $c>0$ and a real number $q>1$ such that $\rho_{E}(\tau) \leq c \tau^{q}$, then $E$ is said to be q-uniformly smooth. Typical examples of such spaces are the $L_{p}, \ell_{p}$ and $W_{p}^{m}$ spaces for $1<p<\infty$ where,

$$
L_{p}\left(\text { or } l_{p}\right) \text { or } W_{p}^{m} \text { is }
$$

$$
\left\{\begin{array}{lll}
2-\text { uniformly smooth and } p \text { - uniformly convex } & \text { if } \quad 2 \leq p<\infty \\
2 \text { - uniformly convex and } p \text { - uniformly smooth } & \text { if } \quad 1<p<2
\end{array}\right.
$$

It is known that a normed linear space $E$ is uniformly smooth if

$$
\lim _{\tau \rightarrow 0} \frac{\rho_{E}(\tau)}{\tau}=0
$$


If there exists a constant $c>0$ and a real number $q>1$ such that $\rho_{E}(\tau) \leq c \tau^{q}$, then $E$ is said to be q-uniformly smooth. Typical examples of such spaces are the $L_{p}, \ell_{p}$ and $W_{p}^{m}$ spaces for $1<p<\infty$ where,

$$
L_{p}\left(\text { or } l_{p}\right) \text { or } W_{p}^{m} \text { is } \begin{cases}2-\text { uniformly smooth and } p \text { - uniformly convex } & \text { if } 2 \leq p<\infty ; \\ 2-\text { uniformly convex and } p \text { - uniformly smooth } & \text { if } 1<p<2 .\end{cases}
$$

Let $J_{q}$ denote the generalized duality mapping from $E$ to $2^{E^{*}}$ defined by

$$
J_{q}(x):=\left\{f \in E^{*}:\langle x, f\rangle=\|x\|^{q} \text { and }\|f\|=\|x\|^{q-1}\right\}
$$

where $\langle.,$.$\rangle denotes the generalized duality pairing. Notice that for x \neq 0$,

$$
J_{q}(x)=\|x\|^{q-2} J_{2}(x), q>1 .
$$

Following Browder [3], we say that a Banach space has a weakly continuous normalized duality map if $J$ is a single-valued and is weak-to-weak ${ }^{*}$ sequentially continous, i.e., if $\left\{x_{n}\right\} \subset E, x_{n} \rightarrow x$, then $J\left(x_{n}\right) \rightarrow J(x)$ in $E^{*}$. Weak continuity of duality map $J$ plays an important role in the fixed point theory for nonlinear operators. Finally recall that a Banach space $E$ satisfies Opial property (see, e.g., [24]) if $\limsup _{n \rightarrow+\infty}\left\|x_{n}-x\right\|<\limsup _{n \rightarrow+\infty}\left\|x_{n}-y\right\|$ whenever $x_{n} \rightarrow x, x \neq y$.

A Banach space E that has a weakly continuous normalized duality map satisfies Opial's property.

Remark 3. Note also that a duality mapping exists in each Banach space. We recall from [1] some of the examples of this mapping in $l_{p}, L_{p}, W^{m, p}$-spaces, $1<p<\infty$.

(i) $l_{p}: J x=\|x\|_{l_{p}}^{2-p} y \in l_{q}, x=\left(x_{1}, x_{2}, \cdots, x_{n}, \cdots\right), y=\left(x_{1}\left|x_{1}\right|^{p-2}, x_{2}\left|x_{2}\right|^{p-2}, \cdots, x_{n}\left|x_{n}\right|^{p-2}, \cdots\right)$,

(ii) $L_{p}: J u=\|u\|_{L_{p}}^{2-p}|u|^{p-2} u \in L_{q}$,

(iii) $W^{m, p}: J u=\|u\|_{W^{m, p}}^{2-p} \sum_{|\alpha \leq m|}(-1)^{|\alpha|} D^{\alpha}\left(\left|D^{\alpha} u\right|^{p-2} D^{\alpha} u\right) \in W^{-m, q}$,

where $1<q<\infty$ is such that $1 / p+1 / q=1$.

Finally recall that a Banach space $E$ satisfies Opial's property (see, e.g., 24]) if $\limsup _{n \rightarrow+\infty}\left\|x_{n}-x\right\|<$ $\limsup _{n \rightarrow+\infty}\left\|x_{n}-y\right\|$ whenever $x_{n} \stackrel{w}{\rightarrow} x, x \neq y$. Recall that an operator $A: K \rightarrow E$ is said to be accretive if there exists $j \in J_{q}(x-y)$ such that

$$
\langle A x-A y, j\rangle \geq 0, \quad \forall x, y \in K
$$


It is said to be strongly accretive if there exists a positive constant $k \in(0,1)$ and such that for all $x, y \in K$, such that

$$
\langle A x-A y, j\rangle \geq k\|x-y\|^{q}, \quad \forall x, y \in K
$$

In a Hilbert space, the normalized duality map is the identity map. Hence, in Hilbert spaces, monotonicity and accretivity coincide. A multi-valued map $A$ defined on a real Banach space $E$ is called $m$-accretive if it is accretive and $R(I+r A)=E$ for some $r>0$ and it is said to satisfy the range condition $R(I+r A)=E$ for all $r>0$.

The operator $A$ in the following example satisfies range condition.

Example 4. Let $A: \mathbb{R} \rightarrow 2^{\mathbb{R}}$ defined by

$$
A x= \begin{cases}\operatorname{sgn}(x), & x \neq 0 \\ {[-1,1],} & x=0\end{cases}
$$

where $A$ is the subdifferential of the absolute value function, $\partial|$.$| , then A$ is $m$-accretive. It can be shown that if $R(I+r A)=E$ for some $r>0$, then this holds for all $r>0$. Hence, m-accretive condition implies range condition.

The demiclosedness of a nonlinear operator $T$ usually plays an important role in dealing with the convergence of fixed point iterative algorithms.

Definition 1. Let $E$ be a real Banach space and $T: D(T) \subset E \rightarrow E$ be a mapping. $I-T$ is said to be demiclosed at 0 if for any sequence $\left\{x_{n}\right\} \subset D(T)$ such that $\left\{x_{n}\right\}$ converges weakly to $p$ and $\left\|x_{n}-T x_{n}\right\|$ converges to zero, then $p \in F(T)$, where $F(T)$ denote the set of fixed points of the mapping $T$.

Lemma 5 (Demiclosedness principle, [3]). Let E be a real Banach space satisfying Opial's property, $K$ be a closed convex subset of $E$, and $T: K \rightarrow K$ be a nonexpansive mapping such that $F(T) \neq \emptyset$. Then $I-T$ is demiclosed; that is,

$$
\left\{x_{n}\right\} \subset K, x_{n} \rightarrow x \in K \text { and }(I-T) x_{n} \rightarrow y \text { implies that }(I-T) x=y
$$

Lemma 6 (22]). Let $E$ be a smooth real Banach space. Then, we have

$$
\|x+y\|^{2} \leq\|x\|^{2}+2\langle y, J(x+y)\rangle \forall x, y \in E .
$$

Lemma 7 ([31] $)$. Assume that $\left\{a_{n}\right\}$ is a sequence of nonnegative real numbers such that $a_{n+1} \leq$ $\left(1-\alpha_{n}\right) a_{n}+\sigma_{n}$ for all $n \geq 0$, where $\left\{\alpha_{n}\right\}$ is a sequence in $(0,1)$ and $\left\{\sigma_{n}\right\}$ is a sequence in $\mathbb{R}$ such that
(a) $\sum_{n=0}^{\infty} \alpha_{n}=\infty$
(b) $\limsup _{n \rightarrow \infty} \frac{\sigma_{n}}{\alpha_{n}} \leq 0$ or $\sum_{n=0}^{\infty}\left|\sigma_{n}\right|<\infty$. Then $\lim _{n \rightarrow \infty} a_{n}=0$. 
Theorem 8. [9] Let $q>1$ be a fixed real number and $E$ be a smooth Banach space. Then the following statements are equivalent:

(i) $E$ is q-uniformly smooth.

(ii) There is a constant $d_{q}>0$ such that for all $x, y \in E$

$$
\|x+y\|^{q} \leq\|x\|^{q}+q\left\langle y, J_{q}(x)\right\rangle+d_{q}\|y\|^{q} .
$$

(iii) There is a constant $c_{1}>0$ such that

$$
\left\langle x-y, J_{q}(x)-J_{q}(y)\right\rangle \leq c_{1}\|x-y\|^{q} \quad \forall x, y \in E .
$$

Lemma 9 ( 8]). Let $E$ be a uniformly convex real Banach space. For arbitrary $r>0$, let $B(0)_{r}:=\{x \in E:\|x\| \leq r\}$, a closed ball with center 0 and radius $r>0$. For any given sequence $\left\{u_{1}, u_{2}, \ldots \ldots, u_{n}, \ldots ..\right\} \subset B(0)_{r}$ and any positive real numbers $\left\{\lambda_{1}, \lambda_{2}, \ldots ., \lambda_{n}, \ldots.\right\}$ with $\sum_{k=1}^{\infty} \lambda_{k}=1$, there exists a continuous, strictly increasing and convex function

$$
g:[0,2 r] \rightarrow \mathbb{R}^{+}, g(0)=0,
$$

such that for any integer $i, j$ with $i<j$,

$$
\left\|\sum_{k=1}^{\infty} \lambda_{k} u_{k}\right\|^{2} \leq \sum_{k=1}^{\infty} \lambda_{k}\left\|u_{k}\right\|^{2}-\lambda_{i} \lambda_{j} g\left(\left\|u_{i}-u_{j}\right\|\right) .
$$

Lemma 10. 333 Let $H$ be a real Hilbert space and $K$ a nonempty, closed convex subset of $H$. Let $A: K \rightarrow H$ be a k-strongly monotone and L-Lipschitzian operator with $k>0, L>0$. Assume that $0<\eta<\frac{2 k}{L^{2}}$ and $\tau=\eta\left(k-\frac{L^{2} \eta}{2}\right)$. Then for each $t \in\left(0, \min \left\{1, \frac{1}{\tau}\right\}\right)$, we have

$$
\|(I-t \eta A) x-(I-t \eta A) y\| \leq(1-t \tau)\|x-y\| \forall x, y \in K .
$$

Let $C$ be a nonempty subsets of a real Banach space $E$. A mapping $Q_{C}: E \rightarrow C$ is said to be sunny if

$$
Q_{C}\left(Q_{C} x+t\left(x-Q_{C} x\right)\right)=Q_{C} x
$$

for each $x \in E$ and $t \geq 0$. A mapping $Q_{C}: E \rightarrow C$ is said to be a retraction if $Q_{C} x=x$ for each $x \in C$.

Lemma 11. [26] Let $C$ and $D$ be nonempty subsets of a smooth real Banach space $E$ with $D \subset C$ and $Q_{D}: C \rightarrow D$ a retraction from $C$ into $D$. Then $Q_{D}$ is sunny and nonexpansive if and only if

$$
\left\langle z-Q_{D} z, J\left(y-Q_{D} z\right)\right\rangle \leq 0
$$

for all $z \in C$ and $y \in D$. 
Remark 12. If $K$ is a nonempty closed convex subset of a Hilbert space $H$, then the nearest point projection $P_{K}$ from $H$ to $K$ is the sunny nonexpansive retraction.

The resolvent operator has the following properties:

Lemma 13. [12] For any $r>0$.

(i) $A$ is accretive if and only if the resolvent $J_{r}^{A}$ of $A$ is single-valued and nonexpansive;

(ii) $A$ is $m$-accretive if and only if $J_{r}^{A}$ of $A$ is single-valued and nonexpansive and its domain is the entire $E$;

(iii) $0 \in A\left(x^{*}\right)$ if and only if $x^{*} \in F\left(J_{r}^{A}\right)$, where $F\left(J_{r}^{A}\right)$ denotes the fixed-point set of $J_{r}^{A}$.

Lemma 14. ([23]) For any $r>0$ and $\mu>0$, the following holds:

$$
\frac{\mu}{r} x+\left(1-\frac{\mu}{r}\right) J_{r}^{A} x \in D\left(J_{r}^{A}\right)
$$

and

$$
J_{r}^{A} x=J_{\mu}^{A}\left(\frac{\mu}{r} x+\left(1-\frac{\mu}{r}\right) J_{r}^{A} x\right) .
$$

Lemma 15. [7] Let $A$ be a continuous accretive operator defined on a real Banach space $E$ with $D(A)=E$. Then $A$ is m-accretive.

\section{Main results}

For our main theorem, we shall need the following lemma.

Lemma 16. Let $q>1$ be a fixed real number and $E$ be a q-uniformly smooth real Banach space with constant $d_{q}$. Let $A: E \rightarrow E$ be a k-strongly accretive and L-Lipschitzian operator with $k>0$, $L>0$. Assume that $\eta \in\left(0, \min \left\{1,\left(\frac{k q}{d_{q} L^{q}}\right)^{\frac{1}{q-1}}\right\}\right)$ and $\tau=\eta\left(k-\frac{d_{q} L^{q} \eta^{q-1}}{q}\right)$. Then for each $t \in\left(0, \min \left\{1, \frac{1}{\tau}\right\}\right)$, we have

$$
\|(I-t \eta A) x-(I-t \eta A) y\| \leq(1-t \tau)\|x-y\|, \forall x, y \in E .
$$

Proof. Without loss of generality, assume $k<\frac{1}{q}$. Then, as $\eta<\left(\frac{k q}{d_{q} L^{q}}\right)^{\frac{1}{q-1}}$, we have $0<q k-$ $d_{q} L^{q} \eta^{q-1}$. Furthermore, from $k<\frac{1}{q}$, we have $q k-d_{q} L^{q} \eta^{q-1}<1$ so that $0<q k-d_{q} L^{q} \eta^{q-1}<1$. By using (ii) of Theorem 8 and properties of $A$, it follows that

$$
\begin{aligned}
\|(I-t \eta A) x-(I-t \eta A) y\|^{q} & \leq\|x-y\|^{q}+q\left\langle t \eta A y-t \eta A x, J_{q}(x-y)\right\rangle+d_{q}\|t \eta A x-t \eta A y\|^{q} \\
& \leq\|x-y\|^{q}-q t \eta\left\langle A x-A y, J_{q}(x-y)\right\rangle+d_{q}(t \eta)^{q}\|A x-A y\|^{q} \\
& \leq\|x-y\|^{q}-q t k \eta\|x-y\|^{q}+d_{q}(L t \eta)^{q}\|x-y\|^{q} \\
& \leq\left(1-q t k \eta+d_{q} L^{q} t^{q} \eta^{q}\right)\|x-y\|^{q} .
\end{aligned}
$$


Therefore

$$
\|(I-t \eta A) x-(I-t \eta A) y\| \leq\left(1-q t k \eta+d_{q} L^{q} t \eta^{q}\right)^{\frac{1}{q}}\|x-y\| .
$$

Using definition of $\tau$, inequality (3.2) and inequality $(1+x)^{s} \leq 1+s x$, for $x>-1$ and $0<s<1$, we have

$$
\begin{aligned}
\|(I-t \eta A) x-(I-t \eta A) y\| & \leq\left(1-t k \eta+\frac{d_{q} L^{q} t \eta^{q}}{q}\right)\|x-y\| \\
& \leq\left(1-t \eta\left(k-\frac{d_{q} L^{q} \eta^{q-1}}{q}\right)\right)\|x-y\| \\
& \leq(1-t \tau)\|x-y\|,
\end{aligned}
$$

which gives us the required result (3.1). This completes the proof.

Remark 17. Lemma 16 is one generalization of Lemma 10 for a Banach space.

We are now in a position to state and prove our main result.

Theorem 18. Let $q>1$ be a fixed real number and $E$ be a q-uniformly smooth and uniformly convex real Banach space having a weakly continuous duality map. Let $K$ be a nonempty, closed and convex subset of $E$ which is a nonexpansive retract of $E$ with $Q_{K}$ as the nonexpansive retraction. Let $\left\{B_{i}\right\}, i \in \mathbb{N}^{*}$ be an infinite family of accretive operators of $E$ such that $F:=\bigcap_{i=1}^{\infty} B_{i}{ }^{-1}(0) \neq \emptyset$ and $\bigcap_{i=1}^{\infty} \overline{D\left(B_{i}\right)} \subset K \subset \bigcap_{i=1}^{\infty} R\left(I+r B_{i}\right)$, for all $r>0$. Let $A: K \rightarrow E$ be a $k$-strongly accretive and L-Lipschitzian operator and $f: K \rightarrow E$ be a b-Lipschitzian mapping with a constant $b \geq 0$. Let $\left\{x_{n}\right\}$ and $\left\{y_{n}\right\}$ be sequences defined iteratively from arbitrary $x_{0} \in K$ by:

$$
\left\{\begin{array}{l}
y_{n}=\beta_{n, 0} x_{n}+\sum_{i=1}^{\infty} \beta_{n, i} J_{r_{n}}^{B_{i}} x_{n}, \\
x_{n+1}=Q_{K}\left(\alpha_{n} \gamma f\left(x_{n}\right)+\left(I-\eta \alpha_{n} A\right) y_{n}\right) .
\end{array}\right.
$$

Let $\left.\left\{r_{n}\right\} \subset\right] 0, \infty\left[,\left\{\beta_{n, i}\right\}\right.$ and $\left\{\alpha_{n}\right\}$ be real sequences in $(0,1)$ satisfying:

(i) $\lim _{n \rightarrow \infty} \alpha_{n}=0 ; \quad$ (ii) $\sum_{n=0}^{\infty} \alpha_{n}=\infty, \sum_{i=0}^{\infty} \beta_{n, i}=1$,

(iii) $\lim _{n \rightarrow \infty} \inf r_{n}>0$, and $\lim _{n \rightarrow \infty} \inf \beta_{n, 0} \beta_{n, i}>0$, for all $i \in \mathbb{N}$.

Assume that $0<\eta<\left(\frac{k q}{d_{q} L^{q}}\right)^{\frac{1}{q-1}}$ and $0<b \gamma<\tau$, where $\tau=\eta\left(k-\frac{d_{q} L^{q} \eta^{q-1}}{q}\right)$. Then the sequence $\left\{x_{n}\right\}$ generated by (3.3) converges strongly to $x^{*} \in F$, which is a unique solution of variational inequality

$$
\left\langle\eta A x^{*}-\gamma f\left(x^{*}\right), J\left(x^{*}-p\right)\right\rangle \leq 0, \quad \forall p \in F .
$$


Proof. First of all, we show that the uniqueness of a solution of the variational inequality (3.4). Suppose both $x^{*} \in F$ and $x^{* *} \in F$ are solutions to (3.4). Then

$$
\left\langle\eta A x^{*}-\gamma f\left(x^{*}\right), J\left(x^{*}-x^{* *}\right)\right\rangle \leq 0
$$

and

$$
\left\langle\eta A x^{* *}-\gamma f\left(x^{* *}\right), J\left(x^{* *}-x^{*}\right)\right\rangle \leq 0 .
$$

Adding up (3.5) and (4.3) yields

$$
\begin{aligned}
&\left\langle\eta A x^{* *}-\eta A x^{*}+\gamma f\left(x^{*}\right)-\gamma f\left(x^{* *}\right), J\left(x^{* *}-x^{*}\right)\right\rangle \leq 0 . \\
& \frac{d_{q} L^{q} \eta^{q-1}}{q}>0 \Longleftrightarrow k-\frac{d_{q} L^{q} \eta^{q-1}}{q}<k \\
& \Longleftrightarrow \eta\left(k-\frac{d_{q} L^{q} \eta^{q-1}}{q}\right)<k \eta \\
& \Longleftrightarrow \tau<k \eta .
\end{aligned}
$$

It follows that

$$
0<b \gamma<\tau<k \eta
$$

Noticing that

$$
\left\langle\eta A x^{* *}-\eta A x^{*}+\gamma f\left(x^{*}\right)-\gamma f\left(x^{* *}\right), J_{\varphi}\left(x^{* *}-x^{*}\right)\right\rangle \geq(k \eta-b \gamma)\left\|x^{*}-x^{* *}\right\|^{2}
$$

which implies that $x^{*}=x^{* *}$ and the uniqueness is proved. Below we use $x^{*}$ to denote the unique solution of (3.4). Without loss of generality, we can assume $\alpha_{n} \in\left(0, \min \left\{1, \frac{1}{\tau}\right\}\right)$.

Now, we prove that the sequences $\left\{x_{n}\right\}$ and $\left\{y_{n}\right\}$ are bounded. Let $p \in F$. Using (3.3) and the fact that $J_{r_{n}}^{B_{i}}$ are nonexpansive, we have

$$
\begin{aligned}
\left\|y_{n}-p\right\| & =\left\|\beta_{n, 0} x_{n}+\sum_{i=1}^{\infty} \beta_{n, i} J_{r_{n}}^{B_{i}} x_{n}-p\right\| \\
& \leq \beta_{n, 0}\left\|x_{n}-p\right\|+\sum_{i=1}^{\infty} \beta_{n, i}\left\|J_{r_{n}}^{B_{i}} x_{n}-p\right\| \\
& \leq \beta_{n, 0}\left\|x_{n}-p\right\|+\sum_{i=1}^{\infty} \beta_{n, i}\left\|x_{n}-p\right\| \\
& \leq\left\|x_{n}-p\right\| .
\end{aligned}
$$


Using Lemma 16, we have

$$
\begin{aligned}
\left\|x_{n+1}-p\right\| & =\left\|Q_{K}\left(\alpha_{n} \gamma f\left(x_{n}\right)+\left(I-\eta \alpha_{n} A\right) y_{n}\right)-p\right\| \\
& \leq\left\|\alpha_{n} \gamma f\left(x_{n}\right)+\left(I-\eta \alpha_{n} A\right) y_{n}-p\right\| \\
& \leq \alpha_{n} \gamma\left\|f\left(x_{n}\right)-f(p)\right\|+\left(1-\tau \alpha_{n}\right)\left\|y_{n}-p\right\|+\alpha_{n}\|\gamma f(p)-\eta A p\| \\
& \leq\left(1-\alpha_{n}(\tau-b \gamma)\right)\left\|x_{n}-p\right\|+\alpha_{n}\|\gamma f(p)-\eta A p\| \\
& \leq \max \left\{\left\|x_{n}-p\right\| \frac{\|\gamma f(p)-\eta A p\|}{\tau-b \gamma}\right\} .
\end{aligned}
$$

By induction, it is easy to see that

$$
\left\|x_{n}-p\right\| \leq \max \left\{\left\|x_{0}-p\right\|, \frac{\|\gamma f(p)-\eta A p\|}{\tau-b \gamma}\right\}, \quad n \geq 1 .
$$

Hence $\left\{x_{n}\right\}$ is bounded also are $\left\{f\left(x_{n}\right)\right\}$, and $\left\{A x_{n}\right\}$.

Let $k \in \mathbb{N}^{*}$, from Lemma 9 and (3.3), we have

$$
\begin{aligned}
\left\|y_{n}-p\right\|^{2} & =\left\|\beta_{n, 0} x_{n}+\sum_{i=1}^{\infty} \beta_{n, i} J_{r_{n}}^{B_{i}} x_{n}-p\right\|^{2} \\
& \leq \beta_{n, 0}\left\|x_{n}-p\right\|^{2}+\sum_{i=1}^{\infty} \beta_{n, i}\left\|J_{r_{n}}^{B_{i}} x_{n}-p\right\|^{2}-\beta_{n, 0} \beta_{n, k} g\left(\left\|J_{r_{n}}^{B_{k}} x_{n}-x_{n}\right\|\right) \\
& \leq\left\|x_{n}-p\right\|^{2}-\beta_{n, 0} \beta_{n, k} g\left(\left\|J_{r_{n}}^{B_{k}} x_{n}-x_{n}\right\|\right) .
\end{aligned}
$$

Consequently, we obtain

$$
\begin{aligned}
\left\|x_{n+1}-p\right\|^{2}= & \left\|Q_{K}\left(\alpha_{n} \gamma f\left(x_{n}\right)+\left(I-\eta \alpha_{n} A\right) y_{n}\right)-p\right\|^{2} \\
\leq & \left\|\alpha_{n}\left(\gamma f\left(x_{n}\right)-\eta A p\right)+\left(I-\eta \alpha_{n} A\right)\left(y_{n}-p\right)\right\|^{2} \\
\leq & \alpha_{n}^{2}\left\|\gamma f\left(x_{n}\right)-\eta A p\right\|^{2}+\left(1-\tau \alpha_{n}\right)^{2}\left\|y_{n}-p\right\|^{2}+2 \alpha_{n}\left(1-\tau \alpha_{n}\right) \| \gamma f\left(x_{n}\right) \\
& -\eta A p\|\| y_{n}-p \| \\
\leq & \alpha_{n}^{2}\left\|\gamma f\left(x_{n}\right)-\eta A p\right\|^{2}+\left(1-\tau \alpha_{n}\right)^{2}\left\|x_{n}-p\right\|^{2}-\left(1-\tau \alpha_{n}\right)^{2} \beta_{n, 0} \beta_{n, k} g\left(\left\|J_{r_{n}}^{B_{k}} x_{n}-x_{n}\right\|\right) \\
& +2 \alpha_{n}\left(1-\tau \alpha_{n}\right)\left\|\gamma f\left(x_{n}\right)-\eta A p\right\|\left\|x_{n}-p\right\|
\end{aligned}
$$

Thus, for every $k \in \mathbb{N}^{*}$, we get

$$
\begin{array}{r}
\left(1-\tau \alpha_{n}\right)^{2} \beta_{n, 0} \beta_{n, k} g\left(\left\|J_{r_{n}}^{B_{k}} x_{n}-x_{n}\right\|\right) \leq\left\|x_{n}-p\right\|^{2}-\left\|x_{n+1}-p\right\|^{2}+\alpha_{n}^{2}\left\|\gamma f\left(x_{n}\right)-\eta A p\right\|^{2} \\
+2 \alpha_{n}\left(1-\tau \alpha_{n}\right)\left\|\gamma f\left(x_{n}\right)-\eta A p\right\|\left\|x_{n}-p\right\| .
\end{array}
$$

Since $\left\{x_{n}\right\}$ and $\left\{f\left(x_{n}\right)\right\}$ are bounded, there exists a constant $C>0$ such that

$$
\left(1-\tau \alpha_{n}\right)^{2} \beta_{n, 0} \beta_{n, k} g\left(\left\|J_{r_{n}}^{B_{k}} x_{n}-x_{n}\right\|\right) \leq\left\|x_{n}-p\right\|^{2}-\left\|x_{n+1}-p\right\|^{2}+\alpha_{n} C .
$$

Let $V I(A, F)$ the solutions set of variational inequality (3.4). Now, we prove $V I(A, F)$ is nonempty. Let $t_{0}$ be a fixed real number such that $t_{0} \in\left(0, \min \left\{1, \frac{1}{\tau}\right\}\right)$. We observe that $Q_{F}\left(I+\left(t_{0} \gamma f-t_{0} \eta A\right)\right)$ 
is a contraction, where $Q_{F}$ is the sunny nonexpansive retraction from $E$ to $F$. Indeed, for all $x, y \in K$, by Lemma [16, we have

$$
\begin{aligned}
\left\|Q_{F}\left(I+\left(t_{0} \gamma f-t_{0} \eta A\right)\right) x-Q_{F}\left(I+\left(t_{0} \gamma f-t_{0} \eta A\right)\right) x\right\| \leq & \|\left(I+\left(t_{0} \gamma f-t_{0} \eta A\right)\right) x \\
& -\left(I+\left(t_{0} \gamma f-t_{0} \eta A\right)\right) x \| \\
\leq & t_{0} \gamma\|f(x)-f(y)\| \\
& +\left\|\left(I-t_{0} \eta A\right) x-\left(I-t_{0} \eta A\right) y\right\| \\
\leq & \left(1-t_{0}(\tau-\gamma)\right)\|x-y\| .
\end{aligned}
$$

Banach's Contraction Mapping Principle guarantees that $Q_{F}\left(I+\left(t_{0} \gamma f-t_{0} \eta A\right)\right)$ has a unique fixed point, say $x_{1} \in E$. That is, $x_{1}=Q_{F}\left(I+\left(t_{0} \gamma f-t_{0} \eta A\right)\right) x_{1}$. Thus, in view of Lemma 11, it is equivalent to the following variational inequality problem

$$
\left\langle\eta A x_{1}-\gamma f\left(x_{1}\right), J\left(x_{1}-p\right)\right\rangle \leq 0, \quad \forall p \in F .
$$

Hence, $x_{1} \in V I(A, F)$. By the uniqueness of the solution of (3.4), we have $x_{1}=x^{*}$.

Next, we prove that $\left\{x_{n}\right\}$ converges strongly to $x^{*}$. We divide the proof into two cases.

Case 1. Assume that the sequence $\left\{\left\|x_{n}-p\right\|\right\}$ is monotonically decreasing. Then $\left\{\left\|x_{n}-p\right\|\right\}$ is convergent. Clearly, we have

$$
\left\|x_{n}-p\right\|^{2}-\left\|x_{n+1}-p\right\|^{2} \rightarrow 0
$$

It then implies from (3.9) that

$$
\lim _{n \rightarrow \infty} \beta_{n, 0} \beta_{n, k} g\left(\left\|J_{r_{n}}^{B_{k}} x_{n}-x_{n}\right\|\right)=0 .
$$

Since $\lim _{n \rightarrow \infty} \inf \beta_{n, 0} \beta_{n, k}>0$ and property of $g$, we have

$$
\lim _{n \rightarrow \infty}\left\|x_{n}-J_{r_{n}}^{B_{k}} x_{n}\right\|=0 .
$$

By using the resolvent identity (Lemma 14), for any $r>0$, we conclude that

$$
\begin{aligned}
\left\|x_{n}-J_{r}^{B_{k}} x_{n}\right\| & \leq\left\|x_{n}-J_{r_{n}}^{B_{k}} x_{n}\right\|+\left\|J_{r_{n}}^{B_{k}} x_{n}-J_{r}^{B_{k}} x_{n}\right\| \\
& \leq\left\|x_{n}-J_{r_{n}}^{B_{k}} x_{n}\right\|+\left\|J_{r}^{B_{k}} x_{n}\left(\frac{r}{r_{n}} x_{n}+\left(1-\frac{r}{r_{n}}\right) J_{r_{n}}^{B_{k}} x_{n}\right)-J_{r}^{B_{k}} x_{n}\right\| \\
& \leq\left\|x_{n}-J_{r_{n}}^{B_{k}} x_{n}\right\|+\left\|\frac{r}{r_{n}} x_{n}+\left(1-\frac{r}{r_{n}}\right) J_{r_{n}}^{B_{k}} x_{n}-x_{n}\right\| \\
& \leq\left\|x_{n}-J_{r_{n}}^{B_{k}} x_{n}\right\|+\left|1-\frac{r}{r_{n}}\right|\left\|J_{r_{n}}^{B_{k}} x_{n}-x_{n}\right\| \rightarrow 0, n \rightarrow \infty, \quad \forall k \in \mathbb{N}^{*} .
\end{aligned}
$$

Hence,

$$
\lim _{n \rightarrow \infty}\left\|x_{n}-J_{r}^{B_{k}} x_{n}\right\|=0 .
$$

We show that $\limsup _{n \rightarrow+\infty}\left\langle\eta A x^{*}-\gamma f\left(x^{*}\right), J\left(x^{*}-x_{n}\right)\right\rangle \leq 0$. Since $E$ is reflexive and $\left\{x_{n}\right\}$ is bounded, there exists a subsequence $\left\{x_{n_{j}}\right\}$ of $\left\{x_{n}\right\}$ such that $\left\{x_{n_{j}}\right\}$ converges weakly to $a$ in $K$ and

$$
\limsup _{n \rightarrow+\infty}\left\langle\eta A x^{*}-\gamma f\left(x^{*}\right), J\left(x^{*}-x_{n}\right)\right\rangle=\lim _{j \rightarrow+\infty}\left\langle\eta A x^{*}-\gamma f\left(x^{*}\right), J\left(x^{*}-x_{n_{j}}\right)\right\rangle .
$$


From (3.12), the fact that $J_{r}^{B_{k}}, k \in \mathbb{N}^{*}$ are nonexpansive and Lemma 5 , we obtain $a \in F$. On the other hand, the assumption that the duality mapping is weakly continuous and the fact that $x^{*} \in V I(A, F)$, we then have

$$
\begin{aligned}
\limsup _{n \rightarrow+\infty}\left\langle\eta A x^{*}-\gamma f\left(x^{*}\right), J\left(x^{*}-x_{n}\right)\right\rangle & =\lim _{j \rightarrow+\infty}\left\langle\eta A x^{*}-\gamma f\left(x^{*}\right), J\left(x^{*}-x_{n_{j}}\right)\right\rangle \\
& =\left\langle\eta A x^{*}-\gamma f\left(x^{*}\right), J\left(x^{*}-a\right)\right\rangle \leq 0 .
\end{aligned}
$$

Finally, we show that $x_{n} \rightarrow x^{*}$. Applying Lemma 6 , we get that

$$
\begin{aligned}
\left\|x_{n+1}-x^{*}\right\|^{2}= & \left\|Q_{K}\left(\alpha_{n} \gamma f\left(x_{n}\right)+\left(I-\eta \alpha_{n} A\right) y_{n}\right)-x^{*}\right\|^{2} \\
\leq & \left\langle\alpha_{n} \gamma f\left(x_{n}\right)+\left(I-\eta \alpha_{n} A\right) y_{n}-x^{*}, J\left(x_{n+1}-x^{*}\right)\right\rangle \\
= & \left\langle\alpha_{n} \gamma f\left(x_{n}\right)+\left(I-\eta \alpha_{n} A\right) y_{n}-x^{*}-\alpha_{n} \gamma f\left(x^{*}\right)+\alpha_{n} \gamma f\left(x^{*}\right)-\alpha_{n} \eta A x^{*}\right. \\
& \left.+\alpha_{n} \eta A x^{*}, J\left(x_{n+1}-x^{*}\right)\right\rangle \\
\leq & \left(\alpha_{n} \gamma\left\|f\left(x_{n}\right)-f\left(x^{*}\right)\right\|+\left\|\left(I-\alpha_{n} \eta A\right)\left(y_{n}-x^{*}\right)\right\|\right)\left\|x_{n+1}-x^{*}\right\| \\
& +\alpha_{n}\left\langle\eta A x^{*}-\gamma f\left(x^{*}\right), J\left(x^{*}-x_{n+1}\right)\right\rangle \\
\leq & \left(1-\alpha_{n}(\tau-b \gamma)\right)\left\|x_{n}-x^{*}\right\|\left\|x_{n+1}-x^{*}\right\|+\alpha_{n}\left\langle\eta A x^{*}-\gamma f\left(x^{*}\right), J\left(x^{*}-x_{n+1}\right)\right\rangle \\
\leq & \left(1-\alpha_{n}(\tau-b \gamma)\right)\left\|x_{n}-x^{*}\right\|^{2}+2 \alpha_{n}\left\langle\eta A x^{*}-\gamma f\left(x^{*}\right), J\left(x^{*}-x_{n+1}\right)\right\rangle .
\end{aligned}
$$

From Lemma 7 , its follows that $x_{n} \rightarrow x^{*}$.

Case 2. Assume that the sequence $\left\{\left\|x_{n}-x^{*}\right\|\right\}$ is not monotonically decreasing. Set $B_{n}=\left\|x_{n}-x^{*}\right\|$ and $\tau: \mathbb{N} \rightarrow \mathbb{N}$ be a mapping for all $n \geq n_{0}$ (for some $n_{0}$ large enough) by $\tau(n)=\max \{k \in \mathbb{N}$ : $\left.k \leq n, B_{k} \leq B_{k+1}\right\}$.

We have $\tau$ is a non-decreasing sequence such that $\tau(n) \rightarrow \infty$ as $n \rightarrow \infty$ and $B_{\tau(n)} \leq B_{\tau(n)+1}$ for $n \geq n_{0}$. Let $i \in \mathbb{N}^{*}$, from (3.9), we have

$$
\left(1-\tau \alpha_{\tau(n)}\right)^{2} \beta_{\tau(n), 0} \beta_{\tau(n), i} g\left(\left\|J_{r_{\tau(n)}}^{B_{i}} x_{\tau(n)}-x_{\tau(n)}\right\|\right) \leq \alpha_{\tau(n)} C \rightarrow 0 \text { as } n \rightarrow \infty .
$$

Furthermore, we have

$$
\beta_{\tau(n), 0} \beta_{\tau(n), i} g\left(\left\|J_{r_{\tau(n)}}^{B_{i}} x_{\tau(n)}-x_{\tau(n)}\right\|\right) \rightarrow 0 \text { as } n \rightarrow \infty .
$$

Hence,

$$
\lim _{n \rightarrow \infty}\left\|J_{r_{\tau(n)}}^{B_{i}} x_{\tau(n)}-x_{\tau(n)}\right\|=0 .
$$

By same argument as in Case 1, we can show that $x_{\tau(n)}$ and $y_{\tau(n)}$ are bounded in $K$ and $\limsup _{\tau(n) \rightarrow+\infty}\left\langle\eta A x^{*}-\gamma f\left(x^{*}\right), J\left(x^{*}-x_{\tau(n)}\right)\right\rangle \leq 0$. We have for all $n \geq n_{0}$,

$0 \leq\left\|x_{\tau(n)+1}-x^{*}\right\|^{2}-\left\|x_{\tau(n)}-x^{*}\right\|^{2} \leq \alpha_{\tau(n)}\left[-(\tau-b \gamma)\left\|x_{\tau(n)}-x^{*}\right\|^{2}+2\left\langle\eta A x^{*}-\gamma f\left(x^{*}\right), J\left(x^{*}-x_{\tau(n)+1}\right)\right\rangle\right]$,

which implies that

$$
\left\|x_{\tau(n)}-x^{*}\right\|^{2} \leq \frac{2}{\tau-b \gamma}\left\langle\eta A x^{*}-\gamma f\left(x^{*}\right), J\left(x^{*}-x_{\tau(n)+1}\right)\right\rangle .
$$


Then, we have

$$
\lim _{n \rightarrow \infty}\left\|x_{\tau(n)}-x^{*}\right\|^{2}=0
$$

Therefore,

$$
\lim _{n \rightarrow \infty} B_{\tau(n)}=\lim _{n \rightarrow \infty} B_{\tau(n)+1}=0 .
$$

Furthermore, for all $n \geq n_{0}$, we have $B_{\tau(n)} \leq B_{\tau(n)+1}$ if $n \neq \tau(n)$ (that is, $n>\tau(n)$ ); because $B_{j}>B_{j+1}$ for $\tau(n)+1 \leq j \leq n$. As a consequence, we have for all $n \geq n_{0}$,

$$
0 \leq B_{n} \leq \max \left\{B_{\tau(n)}, B_{\tau(n)+1}\right\}=B_{\tau(n)+1} .
$$

Hence, $\lim _{n \rightarrow \infty} B_{n}=0$, that is $\left\{x_{n}\right\}$ converges strongly to $x^{*}$. This completes the proof.

As a consequence of Theorem 18, we have the following theorem.

Theorem 19. Let $q>1$ be a fixed real number and $E$ be a q-uniformly smooth and uniformly convex real Banach space having a weakly continuous duality map. Let $\left\{B_{i}\right\}, i \in \mathbb{N}^{*}$ be an infinite family of m-accretive operators of $E$ such that $F:=\bigcap_{i=1}^{\infty} B_{i}{ }^{-1}(0) \neq \emptyset$. Let $A: E \rightarrow E$ be akstrongly accretive and L-Lipschitzian operator and and $f: K \rightarrow E$ be a b-Lipschitzian mapping with a constant $b \geq 0$. Let $\left\{x_{n}\right\}$ and $\left\{y_{n}\right\}$ be sequences defined iteratively from arbitrary $x_{0} \in E$ by:

$$
\left\{\begin{array}{l}
y_{n}=\beta_{n, 0} x_{n}+\sum_{i=1}^{\infty} \beta_{n, i} J_{r_{n}}^{B_{i}} x_{n}, \\
x_{n+1}=\alpha_{n} \gamma f\left(x_{n}\right)+\left(I-\eta \alpha_{n} A\right) y_{n} .
\end{array}\right.
$$

Let $\left.\left\{r_{n}\right\} \subset\right] 0, \infty\left[,\left\{\beta_{n, i}\right\}\right.$ and $\left\{\alpha_{n}\right\}$ be real sequences in $(0,1)$ satisfying:

(i) $\lim _{n \rightarrow \infty} \alpha_{n}=0 ; \quad$ (ii) $\sum_{n=0}^{\infty} \alpha_{n}=\infty, \quad \sum_{i=0}^{\infty} \beta_{n, i}=1$,

(iii) $\lim _{n \rightarrow \infty} \inf r_{n}>0$, and $\lim _{n \rightarrow \infty} \inf \beta_{n, 0} \beta_{n, i}>0, \quad$ for all $i \in \mathbb{N}$.

Assume that $0<\eta<\left(\frac{k q}{d_{q} L^{q}}\right)^{\frac{1}{q-1}}$ and $0<b \gamma<\tau$, where $\tau=\eta\left(k-\frac{d_{q} L^{q} \eta^{q-1}}{q}\right)$. Then the sequence $\left\{x_{n}\right\}$ generated by (3.14) converges strongly to $x^{*} \in F$, which is a unique solution of variational inequality (3.4).

Proof. Since $B_{i}$ are $m$-accretive operators, we conclude that $B_{i}$ are accretive and satisfy the condition $R\left(I+r B_{i}\right)=E$ for all $r>0$. Setting $K=E$ in Theorem 18, we obtain the desired result.

Corollary 1. Let $H$ be a real Hilbert space. Let $K$ be a nonempty, closed and convex subset of $H$. Let $\left\{B_{i}\right\}, i \in \mathbb{N}^{*}$ be an infinite family of monotone operators of $H$ such that $F:=\bigcap_{i=1}^{\infty} B_{i}{ }^{-1}(0) \neq \emptyset$ and $\bigcap_{i=1}^{\infty} \overline{D\left(B_{i}\right)} \subset K \subset \bigcap_{i=1}^{\infty} R\left(I+r B_{i}\right)$, for all $r>0$. Let $A: K \rightarrow H$ be a strongly bounded linear 
operator and and $f: K \rightarrow E$ be a b-Lipschitzian mapping with a constant $b \geq 0$. Let $\left\{x_{n}\right\}$ and $\left\{y_{n}\right\}$ be sequences defined iteratively from arbitrary $x_{0} \in K$ by:

$$
\left\{\begin{array}{l}
y_{n}=\beta_{n, 0} x_{n}+\sum_{i=1}^{\infty} \beta_{n, i} J_{r_{n}}^{B_{i}} x_{n} \\
x_{n+1}=P_{K}\left(\alpha_{n} \gamma f\left(x_{n}\right)+\left(I-\eta \alpha_{n} A\right) y_{n}\right) .
\end{array}\right.
$$

Let $\left.\left\{r_{n}\right\} \subset\right] 0, \infty\left[, \quad\left\{\beta_{n, i}\right\}\right.$ and $\left\{\alpha_{n}\right\}$ be real sequences in $(0,1)$ satisfying:

(i) $\lim _{n \rightarrow \infty} \alpha_{n}=0 ; \quad$ (ii) $\sum_{n=0}^{\infty} \alpha_{n}=\infty, \sum_{i=0}^{\infty} \beta_{n, i}=1$,

(iii) $\lim _{n \rightarrow \infty} \inf r_{n}>0$, and $\lim _{n \rightarrow \infty} \inf \beta_{n, 0} \beta_{n, i}>0$, for all $i \in \mathbb{N}$.

Assume that $0<\eta<\frac{2 k}{\|A\|^{2}}$ and $0<b \gamma<\tau$, where $\tau=\eta\left(k-\frac{\|A\|^{2} \eta}{2}\right)$. Then the sequence $\left\{x_{n}\right\}$ generated by (3.15) converges strongly to $x^{*} \in F$, which is the optimality condition for the minimization problem

$$
\min _{x \in F} \frac{\eta}{2}\langle A x, x\rangle-h(x),
$$

where $h$ is a potential function for $\gamma f$ (i.e. $h^{\prime}(x)=\gamma f(x)$ on $K$ ).

Proof. From Remark 1, we have $A$ is strongly monotone and $\|A\|$-Lipschitz. the proof follows Theorem 18 ,

\section{Applications}

In this section, as applications, we will utilize Theorem 18 to deduced several results. As a direct consequence of Theorem 18, we have the following results:

\subsection{Application to equilibrium problems}

Let $H$ be a real Hilbert space and let $C$ be a nonempty, closed and convex subset of $H$. Let $F$ be a bifunction of $C \times C$ into $\mathbb{R}$, where $\mathbb{R}$ is the real numbers. The equilibrium problem for $F$ is to find $x \in C$ such that

$$
F(x, y) \geq 0, \forall y \in C
$$

The set of solutions is denoted by $E P(F)$. Equilibrium problems which were introduced by Fan [1] and Blum and Oettli [4 have had a great impact and influence on the development of several branches of pure and applied sciences. For solving the equilibrium problem for a bifunction $F: C \times C \rightarrow \mathbb{R}$, let us assume that $f$ satisfies the following conditions:

(A1) $F(x, x)=0$ for all $x \in C$;

$(A 2) F$ is monotone, i.e., $F(x, y)+F(y, x) \leq 0$ for all $x, y \in C$; 
(A3) for each $x, y, z \in C$,

$$
\lim _{t \rightarrow 0} F(t z+(1-t) x, y) \leq F(x, y)
$$

(A4) for each $x \in C, \quad y \rightarrow F(x, y)$ is convex and lower semicontinuous.

Lemma 20. [6] Assume that $F: C \times C \rightarrow \mathbb{R}$ satisfying $(A 1)-(A 4)$. For $r>0$ and $x \in H$, define a mapping $T_{r}: H \rightarrow C$ as follows

$$
T_{r}(x)=\left\{z \in C, F(z, y)+\frac{1}{r}\langle y-z, z-x\rangle \geq 0, \quad \forall y \in C\right\},
$$

for all $x \in H$. Then, the following hold:

1.T $T_{r}$ is single-valued;

2. $T_{r}$ is firmly nonexpansive, i.e., $\left\|T_{r}(x)-T_{r}(y)\right\|^{2} \leq\left\langle T_{r} x-T_{r} y, x-y\right\rangle$ for any $x, y \in H$;

3.F $\left(T_{r}\right)=E P(F)$;

4.EP(F) is closed and convex.

The following lemma appears implicitly in 29 .

Lemma 21. [29] Let $H$ be a Hilbert space and let $C$ be a nonempty closed convex subset of $H$. Let $F: C \times C \rightarrow \mathbb{R}$ satisfy $(A 1)-(A 4)$. Let $A_{F}$ be a set-valued mapping of $H$ into itself defined by:

$$
A_{F} x=\left\{\begin{array}{l}
\{z \in H, F(x, y) \geq\langle y-x, z\rangle, \forall y \in C,\} \quad \forall x \in C \\
\emptyset, \quad x \notin C .
\end{array}\right.
$$

Then $\operatorname{EP}(F)=A_{F}{ }^{-1}(0)$ and $A_{F}$ is a maximal monotone operator with $D\left(A_{F}\right) \subset C$. Furthermore, for any $x \in H$ and $r>0$, the map $T_{r}$ defined as Lemma 20 coincides with the resolvent of $A_{F}$, i.e,

$$
T_{r} x=\left(I+r A_{F}\right)^{-1} x .
$$

Using Theorem 18, we prove a strong convergence theorem for an equilibrium problem in a Hilbert space.

Theorem 22. Let $H$ be a real Hilbert space and $F: H \times H \rightarrow \mathbb{R}$ satisfying (A1)-(A4) such that $E P(F) \neq \emptyset$. Let $A: H \rightarrow H$ be a k-strongly monotone and L-Lipschitzian operator and $f: K \rightarrow E$ be a b-Lipschitzian mapping with a constant $b \geq 0$. Let $\left\{x_{n}\right\},\left\{u_{n}\right\}$ and $\left\{y_{n}\right\}$ be a sequences defined iteratively from arbitrary $x_{0} \in H$ by:

$$
\left\{\begin{array}{l}
F\left(u_{n}, y\right)+\frac{1}{r_{n}}\left\langle y-u_{n}, u_{n}-x_{n}\right\rangle \geq 0, \forall y \in H \\
y_{n}=\beta_{n} x_{n}+\left(1-\beta_{n}\right) u_{n}, \\
x_{n+1}=\alpha_{n} \gamma f\left(x_{n}\right)+\left(I-\eta \alpha_{n} A\right) y_{n} .
\end{array}\right.
$$

Let $\left.\left\{r_{n}\right\} \subset\right] 0, \infty\left[,\left\{\beta_{n}\right\}\right.$ and $\left\{\alpha_{n}\right\}$ be real sequences in $(0,1)$ satisfying:

(i) $\lim _{n \rightarrow \infty} \alpha_{n}=0 ; \quad$ (ii) $\sum_{n=0}^{\infty} \alpha_{n}=\infty, \beta_{n} \in[a, b] \subset(0,1)$. 
(iii) $\lim _{n \rightarrow \infty} \inf r_{n}>0$.

Assume that $0<\eta<\frac{2 k}{L^{2}}$ and $0<b \gamma<\tau$, where $\tau=\eta\left(k-\frac{L^{2} \eta}{2}\right)$. Then the sequence $\left\{x_{n}\right\}$ generated by (4.3) converge strongly to $x^{*} \in E P(f)$, which is a unique solution of variational inequality

$$
\left\langle\eta A x^{*}-\gamma f\left(x^{*}\right), x^{*}-p\right\rangle \leq 0, \quad \forall p \in E P(F)
$$

Proof. Since $F: H \times H \rightarrow \mathbb{R}$ satisfying $(A 1)-(A 4)$, we have that the mapping $A_{F}$ defined by Lemma 21 is a maximal and monotone operator. Put $B=A_{F}$ in Theorem 19](with $\mathrm{i}=1$ ). Then, we obtain that $u_{n}=T_{r_{n}} x_{n}=J_{r_{n}}^{B} x_{n}$. Therefore, we arrive at the desired results.

\subsection{Application to an infinite family of continuous pseudocontractive mappings.}

Let $K$ be a nonempty, closed convex subset of a real Banach space $E$. A mapping $T: K \rightarrow K$ is said to be pseudocontractive if there exists $j(x-y) \in J(x-y)$ such that

$$
\langle T x-T y, j(x-y)\rangle \leq\|x-y\|^{2}, \forall x, y \in K .
$$

It is well known that the class of pseudocontractive mapping is more general than the class of nonexpansive mapping. Moreover, there exists a relationship between the class of accretive mappings and the class of pseudocontractive mappings. A mapping $A: K \rightarrow E$ is said to be pseudocontractive if $T:=I-A$ is accretive. We can observe that $x^{*}$ is a zero of the accretive mapping $A$ if and only if it is a fixed point of the pseudocontractive mapping $T:=I-A$.

Hence, one has the following result.

Theorem 23. Let $q>1$ be a fixed real number and $E$ be a q-uniformly smooth and uniformly convex real Banach space having a weakly continuous duality map. Let $K$ be a nonempty, closed and convex subset of $E$ which is a nonexpansive retract of $E$ with $Q_{K}$ as the nonexpansive retraction. Let $T_{i}: K \rightarrow E, \quad i \in \mathbb{N}^{*}$ be an infinite family of continuous pseudo-contractive mappings of such that $\bigcap_{i=1}^{\infty} F\left(T_{i}\right) \neq \emptyset$. For each $r>0$, let $J_{r}^{i}:=\left(I+r\left(I-T_{i}\right)\right)^{-1}, \quad i \in \mathbb{N}^{*}$. Let $A: K \rightarrow E$ be a $k$-strongly accretive and L-Lipschitzian operator and $f: K \rightarrow E$ be an b-Lipschitzian mapping with a constant $b \geq 0$. Let $\left\{x_{n}\right\}$ and $\left\{y_{n}\right\}$ be sequences defined iteratively from arbitrary $x_{0} \in K$ by:

$$
\left\{\begin{array}{l}
y_{n}=\beta_{n, 0} x_{n}+\sum_{i=1}^{\infty} \beta_{n, i} J_{r_{n}}^{i} x_{n} \\
x_{n+1}=Q_{K}\left(\alpha_{n} \gamma f\left(x_{n}\right)+\left(I-\eta \alpha_{n} A\right) y_{n}\right) .
\end{array}\right.
$$

Let $\left.\left\{r_{n}\right\} \subset\right] 0, \infty\left[, \quad\left\{\beta_{n, i}\right\}\right.$ and $\left\{\alpha_{n}\right\}$ be real sequences in $(0,1)$ satisfying:

(i) $\lim _{n \rightarrow \infty} \alpha_{n}=0 ; \quad$ (ii) $\sum_{n=0}^{\infty} \alpha_{n}=\infty, \sum_{i=0}^{\infty} \beta_{n, i}=1$, 
(iii) $\lim _{n \rightarrow \infty} \inf r_{n}>0$, and $\lim _{n \rightarrow \infty} \inf \beta_{n, 0} \beta_{n, i}>0$, for all $i \in \mathbb{N}^{*}$.

Assume that $0<\eta<\left(\frac{k q}{d_{q} L^{q}}\right)^{\frac{1}{q-1}}$ and $0<b \gamma<\tau$, where $\tau=\eta\left(k-\frac{d_{q} L^{q} \eta^{q-1}}{q}\right)$. Then the sequence $\left\{x_{n}\right\}$ generated by (4.5) converges strongly to $x^{*} \in \bigcap_{i=1}^{\infty} F\left(T_{i}\right)$, which is a unique solution of variational inequality

$$
\left\langle\eta A x^{*}-\gamma f\left(x^{*}\right), J\left(x^{*}-p\right)\right\rangle \leq 0, \quad \forall p \in \bigcap_{i=1}^{\infty} F\left(T_{i}\right) .
$$

Proof. For each $i \in \mathbb{N}^{*}$, we set $B_{i}=I-T_{i}$ into Theorem 18, Then $F\left(T_{i}\right)=B_{i}{ }^{-1}(0)$, for all $i \in \mathbb{N}^{*}$ and hence $\bigcap_{i=1}^{\infty} F\left(T_{i}\right)=\bigcap_{i=1}^{\infty} B_{i}{ }^{-1}(0)$. Furthermore, each $B_{i}$ is $m$-accretive. Therefore, the proof is complete from Theorem 18 . 


\section{References}

[1] Ya. Alber, Metric and generalized Projection Operators in Banach space:properties and applications in Theory and Applications of Nonlinear Operators of Accretive and Monotone Type, (A. G Kartsatos, Ed.), Marcel Dekker, New York, (1996). pp. 15-50.

[2] O. A. Boikanyo and G. Moroşanu, Modified Rockafellar's algorithm, Math. Sci. Res. J. 13 (2009), no. 5, 101-122.

[3] F. E. Browder, Convergenge theorem for sequence of nonlinear operator in Banach spaces, Math.Z.100-201-225, (1976). vol. EVIII, part 2.

[4] E. Blum and W. Oettli, From optimization and variational inequalities to equilibrium problems, Math. Student 63 (1994), no. 1-4, 123-145.

[5] R. E. Bruck, Jr., A strongly convergent iterative solution of $0 \in U(x)$ for a maximal monotone operator $U$ in Hilbert space, J. Math. Anal. Appl. 48 (1974), 114-126.

[6] S. D. Flåm and A. S. Antipin, Equilibrium programming using proximal-like algorithms, Math. Programming 78 (1997), no. 1, Ser. A, 29-41.

[7] I. Cioranescu, Geometry of Banach spaces, duality mappings and nonlinear problems, Mathematics and its Applications, 62, Kluwer Academic Publishers Group, Dordrecht, 1990.

[8] S. Chang, J. K. Kim and X. R. Wang, Modified block iterative algorithm for solving convex feasibility problems in Banach spaces, J. Inequal. Appl. 2010, Art. ID 869684, 14 pp.

[9] C. Chidume, Geometric properties of Banach spaces and nonlinear iterations, Lecture Notes in Mathematics, 1965, Springer-Verlag London, Ltd., London, 2009.

[10] M. Eslamian and J. Vahidi, General proximal point algorithm for monotone operators, Ukraïn. Mat. Zh. 68 (2016), no. 11, 1483-1492.

[11] K. Fan, A minimax inequality and applications, in Inequalities, III, O. Shisha, Ed., (1972). pp. 103-113, Academic Press, New York, NY, USA.

[12] K. Goebel and S. Reich, Uniform convexity, hyperbolic geometry, and nonexpansive mappings, Monographs and Textbooks in Pure and Applied Mathematics, 83, Marcel Dekker, Inc., New York, 1984.

[13] J. K. Kim and T. M. Tuyen, New iterative methods for finding a common zero of a finite family of monotone operators in Hilbert spaces, Bull. Korean Math. Soc. 54 (2017), no. 4, $1347-1359$. 
[14] J. K. Kim and N. Buong, A new iterative method for equilibrium problems and fixed point problems for infinite family of nonself strictly pseudocontractive mappings, Fixed Point Theory Appl. 2013, 2013:286, 13 pp.:10.1186/1687-1812-2013-286

[15] J. K. Kim and T. M. Tuyen, Viscosity approximation method with Meir-Keeler contractions for common zero of accretive operators in Banach spaces, Fixed Point Theory Appl. 2015, 2015:9, 17 pp.

[16] J. K. Kim, P. N. Anh and H. G. Hyun, A proximal point-type algorithm for pseudo equilibrium problems, Bull. Korean Math. Soc., 49 (4)(2012), 747-759.

[17] J.K.Kim and Salahuddin, Existence of solutions for multi-valued equilibrium problems, Nonlinear Funct. Anal. and Appl., 23 (4)(2018), 779-795.

[18] J.K. Kim and Salahuddin, Extragradient methods for generalized mixed equilibrium problems and fixed point problems in Hilbert spaces, Nonlinear Funct. Anal. and Appl., 22(4)(2017), 693-709.

[19] N. Lehdili, A. Moudafi, Combining the proximal algorithm and Tikhonov method, Optimization, 37, (1996). 239-252.

[20] C. Izuchukwu et al., A viscosity iterative technique for equilibrium and fixed point problems in a Hadamard space, Appl. Gen. Topol. 20 (2019), no. 1, 193-210.

[21] L.O. Jolaoso, T.O. Alakoya, A. Taiwo, and O.T. Mewomo, A parallel combination extragradient method with Armijo line searching for finding common solution of finite families of equilibrium and fixed point problems, Rend. Circ. Mat. Palermo II, (2019), DOI:10.1007/s12215019-00431-2

[22] T. C. Lim, H.K. Xu, Fixed point theorems for assymptoticaly nonexpansive mapping, Nonliear Anal 22, no. 11, (1994). 1345-1355.

[23] I. Miyadera, Nonlinear semigroups, Translations of Mathematical Monographs, 109 American Mathematical Society, Providence, RI. (1992).

[24] Z. Opial, Weak convergence of sequence of succecive approximation of nonexpansive mapping, Bull; Amer. Math.soc. 73, (1967). 591-597.

[25] R. T. Rockafellar, Monotone operators and the proximal point algorithm, SIAM J. Control Optim. 14 (1976), no. 5, 877-898.

[26] S. Reich, Weak convergence theorems for nonexpansive mappings in Banach spaces, J. Math. Anal. Appl. 67 (1979), no. 2, 274-276. 
[27] M. V. Solodov and B. F. Svaiter, Forcing strong convergence of proximal point iterations in a Hilbert space, Math. Program. 87 (2000), no. 1, Ser. A, 189-202.

[28] C. Tian and Y. Song, Strong convergence of a regularization method for Rockafellar's proximal point algorithm, J. Global Optim. 55 (2013), no. 4, 831-837.

[29] S. Takahashi, W. Takahashi and M. Toyoda, Strong convergence theorems for maximal monotone operators with nonlinear mappings in Hilbert spaces, J. Optim. Theory Appl. 147 (2010), no. $1,27-41$.

[30] H.-K. Xu, A regularization method for the proximal point algorithm, J. Global Optim. 36 (2006), no. 1, 115-125.

[31] H.-K. Xu, Iterative algorithms for nonlinear operators, J. London Math. Soc. (2) 66 (2002), no. $1,240-256$.

[32] Y. Yao and M. A. Noor, On convergence criteria of generalized proximal point algorithms, J. Comput. Appl. Math. 217 (2008), no. 1, 46-55.

[33] S. Wang, A general iterative method for obtaining an infinite family of strictly pseudocontractive mappings in Hilbert spaces, Appl. Math. Lett. 24 (2011), no. 6, 901-907. 